Sādhanā Vol. 39, Part 2, April 2014, pp. 525-530. C Indian Academy of Sciences

\title{
The stretch zone of automotive steel sheets
}

\author{
L AMBRIŠKO ${ }^{1, *}$ and L PEŠEK ${ }^{2}$ \\ ${ }^{1}$ Institute of Structural Engineering, Faculty of Civil Engineering, \\ Technical University of Košice, Vysokoškolská 4, 04200 Košice, Slovak Republic \\ ${ }^{2}$ Department of Materials Science, Faculty of Metallurgy, \\ Technical University of Košice, Letná 9, 04200 Košice, Slovak Republic \\ e-mail: lubomir.ambrisko@tuke.sk; ladislav.pesek@tuke.sk
}

MS received 22 July 2012; revised 30 July 2013; accepted 10 September 2013

\begin{abstract}
The paper deals with an experimental determination of the stretch zone dimensions in the notch tip in thin steel sheets. The stretch zone dimensions depend on steel grade, on the rolling direction as well as on the loading rate. Stretch zones were observed and measured on three steel grades. Fracture area and stretch zones were analysed by SEM. Stable crack growth was monitored by videoextensometry techniques on CT (Compact Tension) specimens. Specimens were loaded under two loading rates by eccentric tension, whereby the deformation in the notch surrounding area was recorded using a non-contact measurement-videoextensometry technique. Linear relation between the stretch zone dimensions was determined.
\end{abstract}

Keywords. Stretch zone; thin sheet; videoextensometry; CTOD; deformation and fracture; electron microscopy.

\section{Introduction}

The stretch zone (SZ) generates due to plastic deformation, caused by the crack tip blunting. This interaction is demonstrated at a fracture surface as a bounded transition between initiatory crack (e.g., fatigue) and either ductile stable crack growth, or cleavage unstable crack growth. The stretch zone is resulting from an intensive slip (Parilák \& Dojčák 1991), which causes characteristic ductile-steps-relief. Interaction of dislocations with the free surface results in a typical micro-relief (Bassim 1987).

The stretch zone indicates the degree of crack tip blunting which precedes actual crack extension (Chen \& Shi 1990), the scheme of SZ formation is in figure 1. Initiatory crack in unloaded specimen is closed and its surfaces are separated with negligible space (figure 1a).

The crack opens with increasing load (figure 1b) and, consequently, crack tip radius increases (blunting). Increasing the next load causes creation of the voids in the process zone in front of the crack tip. The shape of the crack tip simultaneously changes from round to spiky. Shearing strain in the crack tip area causes crack extension $\Delta a_{S}\left(\Delta a_{S}=w_{S Z}\right.$ the stretch zone width), which still

*For correspondence 

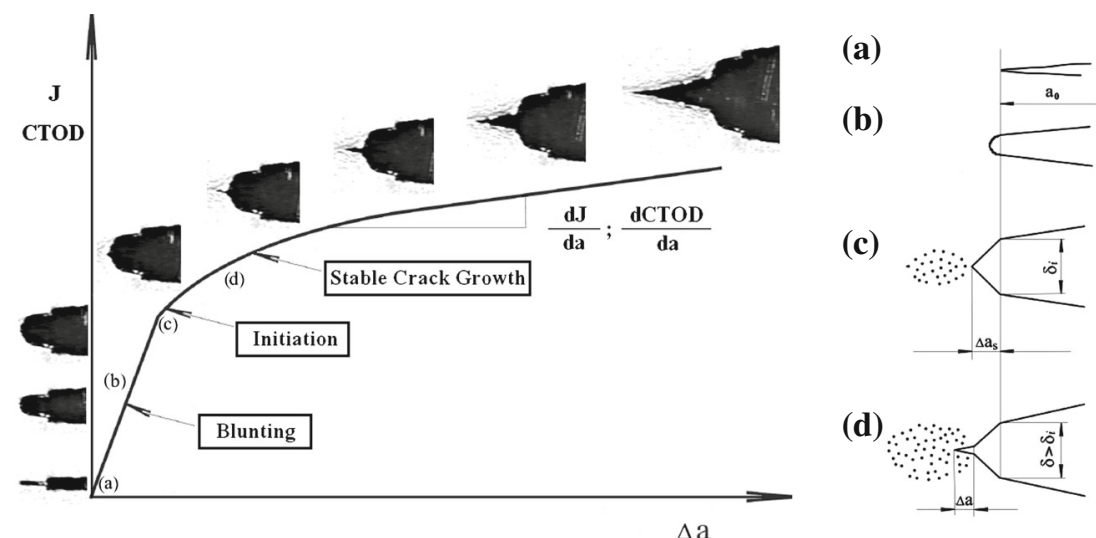

Figure 1. Formation of the stretch zone, (a) unloaded, (b) loaded, crack opening occurs and the stretch zone growths, (c) formation of the voids in front of the crack tip, (d) stable crack growth.

is not a natural crack growth. The opening reaches the value of $\delta_{i}$ for initiation nearly before local tearing of the material in place of highest voids density in the process zone (figure 1c). By the following load increasing, the crack opening $\delta>\delta_{i}$ increases faster, because it is supported by stable crack extension $\Delta a$ (figure 1d), but the size of the stretch zone does not increase during crack propagation (Saxena et al 2009).

Being a precise indication of the extent of the plastic blunting process, the stretch zone width $w_{S Z}$ is recognized as an alternative method for determining the fracture toughness of the material (Tarpani et al 2003). Indeed, it has been very well correlated with critical values of energetic and geometric-based fracture mechanics criteria (Hyatt \& Matthews 1994; Pluvinage \& Lanvin 1993; Putatunda \& Rigsbee 1985). The stretch zone size can be correlated with CTOD (Crack Tip Opening Displacement), suggests that the stretch zone dimensions can be a measure of CTOD (Bassim 1995) and fracture toughness $J_{I C}$ can be correlated with the stretch zone width $w_{S Z}$ (Smith et al 1995). The relationship between the critical CTOD for ductile crack initiation, $\delta_{i}$, with the $w_{S Z}$ (Barnhurst \& Gruzleski 1985; Hopkins \& Jolley 1982) takes the general form of

$$
\delta_{i}=\delta_{0}+\alpha \cdot w_{S Z}
$$

where $\delta_{0}$ is the CTOD at which stretch zones appear on fracture surfaces and is usually considered to be zero; $\alpha$ is a parameter which depends on the geometry of the blunted crack tip, the definition of CTOD and the method for $w_{S Z}$ measurement, the experimental values vary from $\alpha=1$ to $\alpha=20$ (Barnhurst \& Gruzleski 1985).

Relation between CTOD and the stretch zone height $a_{S Z}$ (figure 2) is given by (Yin 1983)

$$
\mathrm{CTOD}=2 \cdot a_{S Z}
$$

The aim of this study is an experimental analysis of the influence of the material, the rolling direction and loading rate on the stretch zone dimensions. Evaluation the stretch zone is enough reworked for bigger thicknesses, but not for thicknesses used for the automotive body (1-2 mm). This study brings new material properties which are necessary for modelling and simulation the crash behaviour of automotive sheets. 


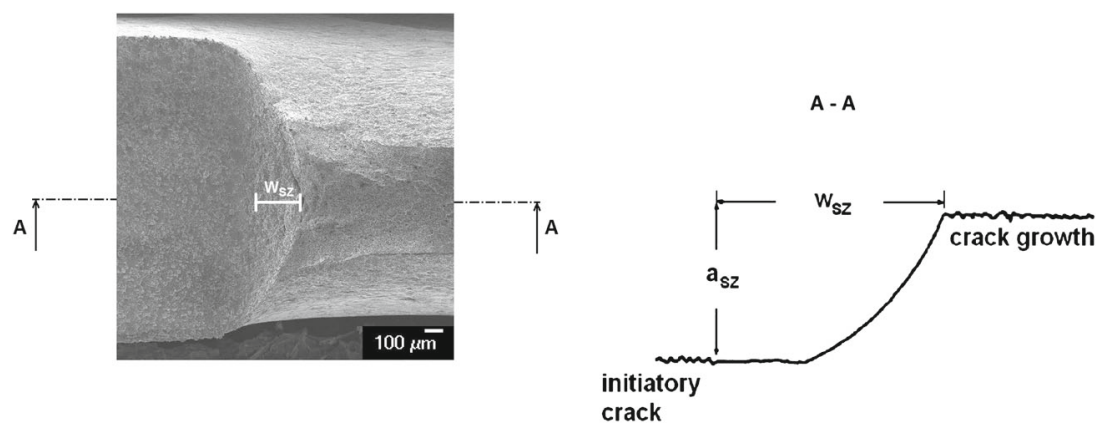

Figure 2. The stretch zone dimensions.

\section{Material and methods}

The materials examined in this study are three grades of thin automotive steel sheets: XSG, HR 45 and DP. (i) XSG is a deep drawing interstitial free steel with ferrite microstructure $(\mathrm{C}=0.0013 \%)$. (ii) HR 45 is a microalloyed steel with ferrite-pearlite microstructure $(\mathrm{C}=$ $0.16 \%)$ and (iii) DP is a dual phase steel with ferrite-martensite microstructure $(\mathrm{C}=0.072 \%)$. Mechanical properties of the investigated steel sheets are in table 1.

Stretch zones were measured for each of the investigated steels; fracture area was observed by JEOL JSM $7000 \mathrm{~F}$ scanning electron microscope. The stable crack growth was monitored using a non-contact videoextensometry technique on electro-spark notched (notch root radius $=$ $0.1 \mathrm{~mm}$ ) CT specimens ( $\mathrm{W}=50 \mathrm{~mm}$ ). The specimen's notch plane was oriented parallel (TL) and perpendicular (LT) to the rolling direction, respectively.

The specimens were loaded by eccentric tension on a tensile testing machine (FP 100/1) at two crosshead-rates: 0.0217 and $2.17 \mathrm{~mm} / \mathrm{s}$. The videoextensometry technique enables us to record displacements and measuring the position of the properly positioned contrast dots. The specimen is illuminated by diffuse light and the specimen surface is recorded with a camera. Notch opening was monitored continuously by recording the co-ordinates of the centre of the gravity of the contrast dots, using the ME-46 Videoextensometer and 'Dot Measuring' software (Spinka 2000). Special software, processing the recorded images, was developed for the SZ width $w_{S Z}$ measuring. The measured notch opening has been recalculated to the CTOD value by the plastic hinge model, used equations are in (BS 5762 1979). The SZ height $a_{S Z}$ using equation (2) was determined from CTOD value. Similarly J-integral values were determined according (ESIS PI-92 1992).

Table 1. Mechanical properties of the investigated steels.

\begin{tabular}{lcccc}
\hline Steel & $\begin{array}{c}\text { Thickness } \\
{[\mathrm{mm}]}\end{array}$ & $\begin{array}{c}\text { Yield } \\
\text { strength } \\
{[\mathrm{MPa}]}\end{array}$ & $\begin{array}{c}\text { Tensile } \\
\text { strength } \\
{[\mathrm{MPa}]}\end{array}$ & $\begin{array}{c}\text { Elongation } \\
\text { (GL: 80 mm) } \\
{[\%]}\end{array}$ \\
\hline XSG & 1.95 & 177 & 286 & 47.2 \\
HR 45 & 1.80 & 360 & 449 & 27 \\
DP & 1.60 & 380 & 576 & 26.2 \\
\hline
\end{tabular}




\section{Results and discussion}

Effect of selected parameters on the stretch zone size was investigated. Figure 3 shows the fracture surface in the area of the SZ for three investigated steel sheets. Evidently, the largest SZ has XSG steel. The DP steel has a bit larger SZ than HR 45 steel grade.

The stretch zone dimensions depend on both the crack orientation to the rolling direction and loading rate, figures 4 and 5. Between the $w_{S Z}$ and CTOD exists a direct relationship (Broek 1974), which also confirms determined linear relation, figure 4. A linear relation was also determined between the stretch zone dimensions $w_{S Z}$ and $a_{S Z}$, figure 5 .

Both SZ dimensions, $w_{S Z}$ and $a_{S Z}$, increase with increasing both CTOD and J-integral. The values $w_{S Z}$ as well $a_{S Z}$ in case of the crack growth in direction perpendicular to the rolling direction (LT) are larger as by the crack growth in the rolling direction (TL) for both loading rates, $0.0217 \mathrm{~mm} / \mathrm{s}$ and $2.17 \mathrm{~mm} / \mathrm{s}$, with an exception of XSG grade.

Differences between LT and TL orientation in tensile properties of investigated steels for both loading rates are within $6 \%$ scatter.

J-integral for both loading rates and for both HR 45 and DP steel reaches higher values by the crack growth in LT orientation. The measured SZ dimensions for higher loading rate $(2.17 \mathrm{~mm} / \mathrm{s})$ are larger than that for lower $(0.0217 \mathrm{~mm} / \mathrm{s})$, with exception of $w_{S Z}$ of HR 45 . By

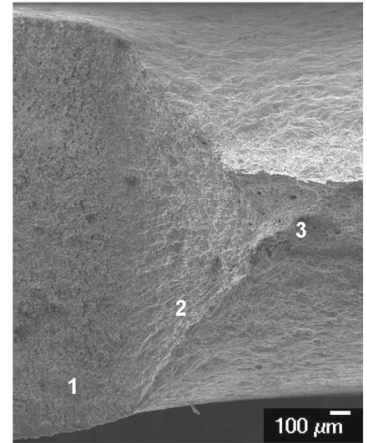

(a)

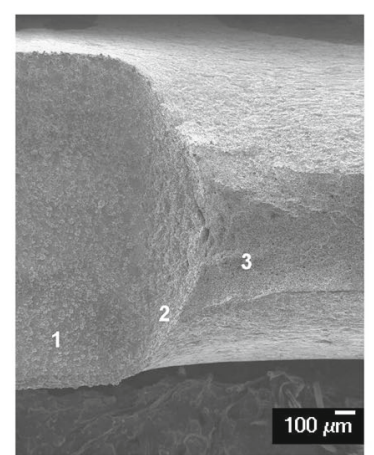

(b)

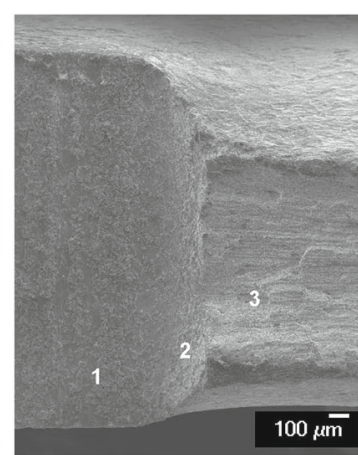

(c)

Figure 3. The stretch zone of the investigated steels. (a) XSG, (b) DP and (c) HR 45 (1 - electro-spark prepared notch, 2 - stretch zone, 3 - stable crack extension).

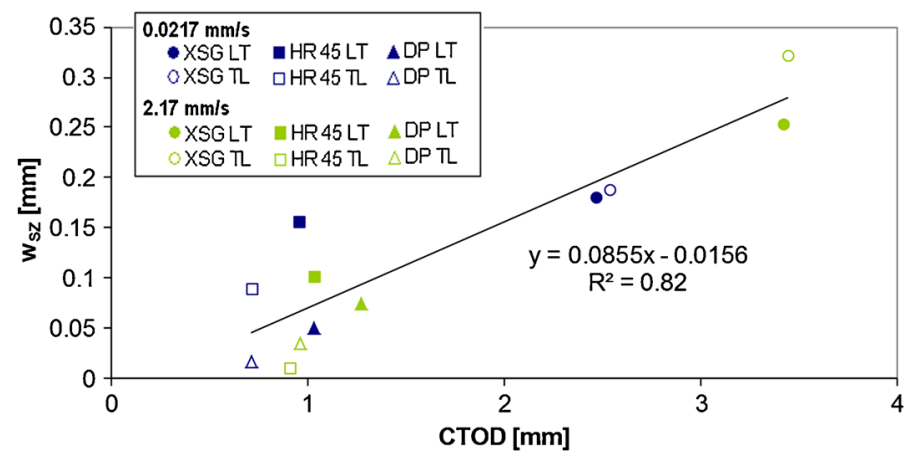

Figure 4. Relation between the stretch zone width $w_{S Z}$ and CTOD. 


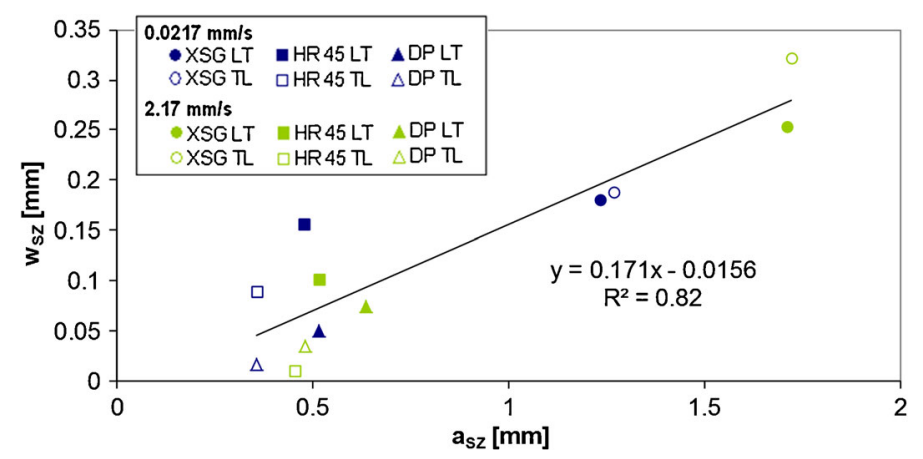

Figure 5. Relation between the stretch zone width $w_{S Z}$ and height $a_{S Z}$.

higher loading rate tensile properties differ for XSG steel by 7-15\% and for both steels HR 45 and DP by $3.5-4.5 \%$ in comparison with lower loading rate.

\section{Conclusions}

The stretch zone dimensions depend on the steel grade as well as on the crack orientation to the rolling direction. The effect of the loading rate is irrelevant for both steels HR 45 and DP.

XSG (IF steel) is most sensitive to influences investigated in this study. The stretch zone of XSG steel is largest in width and height, in comparison with the stretch zone of HR 45 and DP steel grade. By higher loading rate $(2.17 \mathrm{~mm} / \mathrm{s})$ both width and height of the stretch zone reaches higher values in comparison with lower loading rate $(0.0217 \mathrm{~mm} / \mathrm{s})$ for steels XSG and DP.

\section{Acknowledgement}

This work was supported by APVV Project No. APVV-0326-07.

\section{List of symbols}

$a_{S Z}$ stretch zone height

CTOD crack tip opening displacement

$G L$ gauge length

$J_{I C}$ fracture toughness

$w_{S Z}$ stretch zone width

$\Delta a$ stable crack extension

$\Delta a_{s}$ stretch zone width

$\delta$ crack opening

$\delta_{i}$ crack opening for crack initiation

$\delta_{0}$ crack opening for stretch zone formation

\section{References}

Barnhurst R J and Gruzleski J E 1985 Fracture toughness and its development in high purity cast carbon and low alloy steels. Metall. Trans A. 16(4): 613-622 
Bassim M N 1987 The role of dislocations in the fracture of prestrained AISI 4340 steel. Mater. Sci. Eng. 95: 199-207

Bassim M N 1995 Use of the stretch zone for the characterization of ductile fracture. J. Mater. Proc. Technol. 54: 109-113

Broek D 1974 Correlation between stretched zone size and fracture toughness. Eng. Fract. Mech. 6: $173-181$

BS 57621979 Methods for Crack Opening Displacement (COD) Testing

Chen B Y and Shi Y W 1990 A comparison of various dynamic elastoplastic fracture toughness evaluating procedure by instrumented impact test. Eng. Fract. Mech. 36(1): 17-26

ESIS PI-92 1992 Recommendations for Determining the Fracture Resistance of Ductile Materials

Hopkins P and Jolley G 1982 The significance of crack tip blunting (stretch zones) in fracture toughness specimens, in: The Proceedings of the 4th European Conference on Fracture - ECF4, Leoben, Austria, 1: 3

Hyatt C V and Matthews J R 1994 Variation of stretch zone width with J, loading rate, temperature and pre-crack depth. Int. J. Fract. 66: 19-32

Parilák L and Dojč́k J 1991 Fracture mechanics, process zone and microstructure. ICM 6, Kyoto, Japan

Pluvinage G and Lanvin A 1993 Stretch zone geometrical measurement, a particular way to measure fracture toughness. Fatigue Fract. Eng. Mater. Struct. 16: 955-972

Putatunda S K and Rigsbee J M 1985 Influence of specimen size on the crack-opening stretch zone. Mater. Sci. Eng. 70: 111-122

Saxena S, Ramakrishnan N and Dutta B K 2009 Determination of stretch zone width using fem. Eng. Fract. Mech. 76: 911-920

Smith J, Bassim M N and Liu C D 1995 Effects of fatigue precracking on stretch zone formation. Eng. Fract. Mech. 52: 401-408

Spinka O 2000 Videoextensometer ME46. Messphysik Laborgeräte, GmbH, Fürstenfeld Austria

Tarpani J R, Bose W W and Spinelli D 2003 Backscattered electron microscopy technique enhancing stretch zone width imaging for initiation fracture toughness measurements. Mater. Charact. 51: 159-170

Yin S W 1983 An ivestigation of the blunting line. Eng. Fract. Mech. 18: 1025-1036 